\title{
A Mindfulness-Based Program Improves Health in Caregivers of People with Autism Spectrum Disorder: a Pilot Study
}

Article in Mindfulness · July 2014

Impact Factor: $3.69 \cdot$ DOI: $10.1007 /$ s12671-014-0316-0

CITATIONS

4

5 authors, including:

Nicolás Ruiz Robledillo

University of Valencia

23 PUBLICATIONS 69 CITATIONS

SEE PROFILE

\section{Josefa Perez-Blasco}

University of Valencia

14 PUBLICATIONS 42 CITATIONS

SEE PROFILE
Patricia Sariñana-González

University of Valencia

18 PUBLICATIONS 60 CITATIONS

SEE PROFILE

Luis Moya-Albiol

University of Valencia

132 PUBLICATIONS 785 CITATIONS

SEE PROFILE 


\title{
A Mindfulness-Based Program Improves Health in Caregivers of People with Autism Spectrum Disorder: a Pilot Study
}

\author{
Nicolás Ruiz-Robledillo • Patricia Sariñana-González • \\ Josefa Pérez-Blasco • Esperanza González-Bono • \\ Luis Moya-Albiol
}

Published online: 1 July 2014

(C) Springer Science+Business Media New York 2014

\begin{abstract}
Mindfulness-based interventions have demonstrated to be effective in reducing stress and health complaints in clinical populations. However, to our knowledge, biological health markers have not been used in studies of the effectiveness of mindfulness programs in caregivers of people with autism spectrum disorders (ASDs). This study aimed to assess the effects of a mindfulness intervention on mood disturbances and health complaints in this population compared with non-caregivers. The design of the study was quasiexperimental, with repeated measures. Self-reported health, cortisol awakening response (CAR), and afternoon cortisol levels before and after a mindfulness session were assessed at the beginning, middle, and end of the intervention. There was a significant reduction in mood disturbances and afternoon cortisol levels during the sessions in all participants, with the reductions being more pronounced in caregivers. Moreover, all participants showed fewer depressive and somatic symptoms at the end of the program, with an improvement in their self-perceived general health. Nevertheless, the CAR levels had not changed significantly after the program. Overall, these results indicate that mindfulness group therapy could be effective for reducing health complaints and reinforce the validity of these programs for caregivers.
\end{abstract}

Keywords Mindfulness $\cdot$ Health $\cdot$ Caregiver $\cdot$ Autism spectrum disorder $\cdot$ Cortisol

\footnotetext{
N. Ruiz-Robledillo $\cdot$ P. Sariñana-González $\cdot$ E. González-Bono • L. Moya-Albiol $(\square)$

Department of Psychobiology, University of Valencia, Avenida Blasco Ibañez, 21, 46020 Valencia, Spain

e-mail: Luis.Moya@uv.es

J. Pérez-Blasco

Department of Evolutionary and Educational Psychology, University of Valencia, Avenida Blasco Ibañez, 21, 46020 Valencia, Spain
}

\section{Introduction}

The negative consequences of caring for a relative with a diagnosis of autism spectrum disorder (ASD) have been widely described in previous research (De Andrés-García et al. 2012; Ruiz-Robledillo and Moya-Albiol 2013; RuizRobledillo et al. 2014). Caring for such a relative is associated with high levels of burden, depression, anxiety, and poorer self-perceived general health (Ruiz-Robledillo and MoyaAlbiol 2013). Although previous research has highlighted this issue, few studies have assessed the effectiveness of psychotherapeutic intervention programs in reducing self-perceived stress and health complaints in caregivers. Most studies reported to date have implemented educational interventions for training parents how to manage the behavioral problems of offspring (Singer et al. 2007). However, there is some evidence that behavioral-cognitive or multi-component programs oriented to caregivers have positive results, reducing depressive symptomatology and self-perceived stress (Kirkham 1993; Nixon and Singer 1993). That is, although this type of intervention could benefit caregivers, little is known about the efficacy of new therapeutic approaches, such as mindfulness interventions, in caregivers of people with ASD.

Mindfulness interventions have been implemented with excellent results in diverse samples of informal caregivers (Birnie et al. 2010; Franco et al. 2010; Oken et al. 2010). To our knowledge, only three studies have analyzed the effects of mindfulness interventions in caregivers of people with ASDs (Ferraioli and Harris 2012; Singh et al. 2006, 2007). Moreover, only one of them analyzed stress and overall health in caregivers, showing an improvement in both outcome measures at the end of the program (Ferraioli and Harris 2012). In the other two studies, only variables related to the care recipient were evaluated and it was found 
that a mindfulness intervention for parents reduced disruptive behavior in offspring.

Further, few studies have employed biological markers to analyze the benefits of this type of intervention and most of these studies have been carried out with cancer patients and their caregivers (Bränström et al. 2013; Carlson et al. 2007). In cancer patients, mindfulness interventions have been observed to have a modifying effect by producing a decrease in morning cortisol levels in patients with high initial levels and an increase in morning cortisol in patients with lower initial levels (Bränström et al. 2013). With regard to pro-inflammatory biomarkers, a progressive reduction in Th1 cytokine levels was found over 1 year of participation in this type of intervention (Carlson et al. 2007). In caregivers of people with cancer, the participation in a mindfulness-based stress reduction (MBSR) program reduced cortisol and interleukin-6 levels (Lengacher et al. 2012).

As well as showing a benefit in clinical samples, mindfulness interventions have been reported to produce increases in positive affect and reductions in anger, rumination, depression, and anxiety in the general population (Keng et al. 2011). These results indicate that mindfulness interventions are very useful in both clinical and non-clinical samples, reducing health complaints and increasing positive affect. However, this type of intervention has shown to be particularly effective in several samples of people under chronic stress conditions, such as chronic disease, burnout, or caring for people with chronic conditions (Goodman and Schorling 2012; Merkes 2010; Minor et al. 2006). Some authors have proposed an improvement in coping with the stressors and an increase in trait mindfulness as main explanations of the positive effects of this intervention in chronically stressed populations ( et al. 2011; Shapiro et al. 2006).

To our knowledge, no studies have investigated the effects of mindfulness interventions on health in caregivers of people with ASDs using biological markers. As each chronic psychological disorder has its own characteristics and differently affects caregivers, it is necessary to assess the effects of mindfulness programs in this specific population. Biological markers of stress provide objective evidence of the effectiveness of psychological interventions for minimizing health complaints. Their use could reinforce previously obtained results in studies employing self-reported measures, which may be more subjective and less reliable than biological indicators.

Given this, we aimed to analyze the effects of a mindfulness-based program (MBP) on mood state and health complaints through the use of self-reported measures and biological markers of stress, namely afternoon cortisol and cortisol awakening response (CAR), in a sample of parents of individuals with ASDs (caregivers) and parents of typically developing children (non-caregivers). In addition, the efficacy of the program for improving health and negative mood in each group was analyzed comparing caregivers and noncaregivers. We hypothesized that both caregivers and noncaregivers would show better mood (less anxiety, negative mood, and feelings of anger) (Lykins and Baer 2009), as well as lower afternoon cortisol levels (Lengacher et al. 2012), after each evaluated session of MBP. In addition, we hypothesized that the health status of caregivers and non-caregivers would improve after the intervention program, together with an adjustment in morning cortisol levels (Bränström et al. 2013). Finally, we hypothesized that these improvements in health and mood state would be more pronounced in caregivers than non-caregivers after the program. We expected to find this greater improvement in health in caregivers, taking into account that they are a chronically stressed population, and the MBP program is primarily focused on stress management. This hypothesis is in line with the results of a previous research in which this type of program has been used with caregivers (Lengacher et al. 2012; Minor et al. 2006).

\section{Method}

\section{Participants}

The sample was composed of 13 participants: six parents of adolescents with a diagnosis of ASD (one man and five women) and seven parents of age-matched typically developing adolescents (seven women). The inclusion criteria for the experimental group were as follows: being a first-degree relative of an individual with a clinical diagnosis of an ASD (all participants were in fact parents of the care recipient), living at home with the care recipient, and being the main caregiver for at least 2 years before the study. The inclusion criteria for the parents of the control group were having healthy offspring with no chronic illnesses and not having been caregivers to any ill relative or exposed to other chronically stressful situations in the previous 2 years.

All participants participated voluntarily in the study and completed an informed consent form in accordance with the ethical standards of human research (Declaration of Helsinki).

\section{Procedure}

Caregivers were mainly recruited from members of an association of relatives of people with ASD. Firstly, a meeting was conducted with caregivers to explain the aim of the research and the criteria for participation. In this meeting, participants were informed about the contents and procedure of the MBP. After indicating that they wished to participate in the study, selected participants were interviewed to obtain information about the demographic variables and information about their caregiving status (years of caregiving since the definitive diagnosis of their son/daughter, hours per week caregiving), 
care recipient characteristics (severity of autistic symptoms and dependency level), and caregiver burden. A similar meeting and interviews were held for candidates for the control group, in which data were collected for the same variables except those referring to care status.

\section{Mindfulness-Based Program}

The intervention program was run in a group format with nine sessions: first, eight consecutive fortnightly sessions were applied, and then an interval between the eighth and the last session of 2 weeks. The contents of the program in each session are explained below. During the first session, participants were introduced to the contents and the dynamics of the program, the principles underlying the automatic pilot were explained, and a short meditation was conducted. Homework for that week was practicing mindful activity, mindful eating, and short breath meditation. In the second session, participants practiced body scan mediation and discussed dealing with barriers and the principles of the cognitive model of mindfulness. Homework for that week was practicing body scan meditation, mindful activity, and mindful eating. The third session was employed to underline the importance of recalling pleasant events and of differentiating between thoughts and facts, and to practice breathing meditation. Alternate body scan practice with breath mediation and record agreeable events were exercises recommended for practicing at home. In the fourth session, long sitting meditation was practiced and topics concerning stress, reactivity, and parenting were introduced for discussion. Homework was alternate the practice of sitting meditation and lake meditation and $3 \mathrm{~min}$ of breath meditation at fixed times. In the fifth session, nature sound meditation was practiced and discussion was established about acceptance and tolerance. Homework for that week was alternate sounds landscape, the lake meditation and sitting meditation, and 3-min breathing at fixed times and in difficult times. The sixth session was dedicated to explaining walking meditation and discussing the concepts of emotional debt and spaciousness. Homework for that week was the same as that of the past session, including the practice of walking meditation. The seventh session included metta and mountain meditation and discussion of compassion, self-compassion, and self-care. The practice of each type of meditation and metta meditation was the homework for that week. Furthermore, each participant addressed a self-care plan with all of the skills learned during the intervention. In the eighth session, a general review was conducted of the program's contents with short metta and body scan meditations. The last session was based on the practice of metta meditation and meditation without object, as well as closure of the program. As has been indicated, several exercises were prescribed to participants to practice between sessions and the use of these exercises was recapped in each session. All sessions lasted approximately
$2 \mathrm{~h}$ and were conducted between 4:00 and 7:00 pm to control for diurnal variations in cortisol secretion (Dickmeis 2009). This MBP was developed and applied by a specialist and experienced therapist in mindfulness approach interventions since 8 years ago approximately. In this regard, a psychotherapist has been trained in the application of the MBSR program and other types of meditation such as Vipasana meditation. As a specialist, she is a recognized mindfulness therapist from different national and international mindfulness-specialized associations. She has taught several mindfulness courses for clinicians and for patients with chronic conditions, bereaved people, or breastfeeding mothers (Pérez-Blasco et al. 2013).

\section{Evaluation Procedure}

During the program, mood states, self-reported health, and salivary cortisol (Csal) were evaluated at the time of three sessions: session 1 (pre-treatment), session 5 (mid-treatment), and session 9 (post-treatment). The methodology of the evaluation of Csal measures consisted in the collection of five saliva samples (two samples before the session, "pre" and "pre2," and single samples immediately after the session, "post," and 15 and 30 min later). For the analysis, the mean of the first two Csal measurements (pre and pre2) was employed. In addition, each participant completed three mood questionnaires (to assess anxiety, anger, and mood; see below) before and after the aforementioned sessions. Finally, selfreported health (depressive symptomatology, somatic symptoms, and self-perceived general health) was also evaluated after each of these sessions. Before each measurement of biological samples, participants were called by telephone and instructed to abstain from eating, drinking stimulants (such as tea, coffee, or alcohol), brushing their teeth, or smoking during the 2 -h period before the session. The procedure in the evaluation process during the intervention is summarized in Table 1.

\section{Measures}

\section{Mood Profiles}

State anxiety was evaluated using the Spanish version of the State-Trait Anxiety Inventory (STAI-S) (Spielberger et al. 1983; Seisdedos, N. 1982). This inventory is composed of 20 items ranked on a four-point Likert scale from 1 (nothing) to 4 (plenty) examining how participants feel at that moment. The reliability coefficient is 0.62 .

Secondly, mood was evaluated using the abbreviated version of the Profile of Mood States (POMS), developed by Fuentes et al. (1995). This version is composed of 29 items grouped in five subscales: tension, depression, anger, vigor, and fatigue. The total score was also calculated by summing scores on all subscales and subtracting the vigor scale. The 
Table 1 Evaluation times during the application of the MBSR program

\begin{tabular}{|c|c|c|c|c|c|c|c|c|c|}
\hline & Pre-treatment & & & & Mid-treatment & & & & Post-treatment \\
\hline Time & Pre-evaluation & & & & Pre-evaluation & & & & Pre-evaluation \\
\hline \multirow{5}{*}{$\begin{array}{l}15 \text { min before } \\
\text { session }\end{array}$} & Mood profiles & & & & Mood profiles & & & & Mood profiles \\
\hline & STAI-S & & & & STAI-S & & & & STAI-S \\
\hline & POMS & & & & POMS & & & & POMS \\
\hline & STAXI-2 & & & & STAXI-2 & & & & STAXI-2 \\
\hline & $\begin{array}{l}\text { Cortisol sample } \\
\text { pre-1 }\end{array}$ & & & & $\begin{array}{l}\text { Cortisol sample } \\
\text { pre-1 }\end{array}$ & & & & Cortisol sample pre-1 \\
\hline $\begin{array}{l}5 \text { min before } \\
\text { session }\end{array}$ & $\begin{array}{l}\text { Cortisol sample } \\
\text { pre-2 }\end{array}$ & & & & $\begin{array}{l}\text { Cortisol sample } \\
\text { pre-2 }\end{array}$ & & & & Cortisol sample pre- 2 \\
\hline & Session 1 & Session 2 & Session 3 & Session 4 & Session 5 & Session 6 & Session 7 & Session 8 & Session 9 \\
\hline & Post-evaluation & & & & Post-evaluation & & & & Post-evaluation \\
\hline \multirow{5}{*}{$\begin{array}{l}0 \text { min after } \\
\text { session }\end{array}$} & Mood profiles & & & & Mood profiles & & & & Mood profiles \\
\hline & STAI-S & & & & STAI-S & & & & STAI-S \\
\hline & POMS & & & & POMS & & & & POMS \\
\hline & STAXI-2 & & & & STAXI-2 & & & & STAXI-2 \\
\hline & $\begin{array}{l}\text { Cortisol sample } \\
\text { post-3 }\end{array}$ & & & & $\begin{array}{l}\text { Cortisol sample } \\
\text { post-3 }\end{array}$ & & & & Cortisol sample post-3 \\
\hline $\begin{array}{l}15 \text { min after } \\
\text { session }\end{array}$ & $\begin{array}{l}\text { Cortisol sample } \\
\text { post-4 }\end{array}$ & & & & $\begin{array}{l}\text { Cortisol sample } \\
\text { post- } 4\end{array}$ & & & & Cortisol sample post- 4 \\
\hline \multirow{5}{*}{$\begin{array}{l}30 \text { min after } \\
\text { session }\end{array}$} & Health status & & & & Health status & & & & Health status \\
\hline & ESS-R & & & & ESS-R & & & & ESS-R \\
\hline & GHQ & & & & GHQ & & & & GHQ \\
\hline & BDI & & & & BDI & & & & BDI \\
\hline & $\begin{array}{l}\text { Cortisol sample } \\
\text { post-5 }\end{array}$ & & & & $\begin{array}{l}\text { Cortisol sample } \\
\text { post-5 }\end{array}$ & & & & Cortisol sample post- 5 \\
\hline
\end{tabular}

higher the total score, the worse the mood. Cronbach's alpha of this instrument is higher than 0.80 .

Lastly, a Spanish version of the State-Trait Anger Expression Inventory-2 (STAXI-2) (Miguel-Tobal et al. 2001) was used for measuring state anger. It contains 15 items ranked on a four-point Likert scale and distributed into three subscales: feelings, verbal, and physical expression of anger. Cronbach's alpha ranged from 0.67 to 0.89 .

\section{Self-reported Health}

Somatic symptoms were assessed with the Spanish revised version of the Somatic Symptoms Scale (ESS-R) created by Sandín and Chorot (1995). This instrument lists referred symptoms over the last 2 years and is composed of 80 items focused on immunological, cardiovascular, respiratory, gastrointestinal, neurosensory, muscular, dermatological, and genital-urinary complaints, as well as female reproductive system complaints in the case of women. The total score of symptoms is also provided. Each scale is composed of ten items ranked on a five-point Likert scale from 0 , "never," to 4 , "more than five times in the last 2 years," with reliability coefficients ranging from 0.79 to 0.84 .
Perceived general health was assessed with a shorter 28item version of the General Health Questionnaire (GHQ-28) designed by Goldberg and Hillier (1979). Responses are scored on a four-point Likert scale from 0, "better than usual," to 3, "worse than usual." The items are grouped into four scales: somatic symptoms, anxiety and insomnia, social dysfunction, and severe depression, and there is also a total score of self-perceived general health, all having Cronbach's alpha higher than 0.92 .

Depressive symptomatology was evaluated by the Beck Depression Inventory (BDI, Beck and Steer 1993). This questionnaire consists of 21 self-report items that refer to emotional, cognitive, and somatic aspects of depression mood. Each item is ranked on a four-point Likert scale and evaluates intensity and severity of symptoms with a reliability coefficient of 0.83 .

\section{Cortisol Measures}

Salivette tubes and a dental cotton roll (Sarstedt, Rommelsdorf, Germany) were employed to collect saliva samples for both afternoon cortisol and CAR. In both cases, participants were instructed to abstain from eating, drinking, smoking, or brushing their teeth before collecting the samples, 
as noted above. Moreover, in the case of samples of CAR, participants were instructed how to store saliva samples and record the exact time of saliva collection. CAR was assessed on two consecutive days using four saliva samples. Researchers provided verbal and written instructions about the details concerning the collection of the first saliva sample immediately after awakening and subsequent samples 30,45 , and 60 min later (samples 1, 2, 3, and 4, respectively). To encourage and assess adherence to the sampling protocol, participants were asked to complete a time sheet recording when they woke up and when they collected the saliva samples. The CAR was calculated as an average salivary cortisol level at each of the time points over two consecutive days. In addition, participants were asked to take note of their level of energy, expectations about the day, and other variables such as consumption of stimulants or alcohol the day before, any smoking the day before, and their number of hours of sleep.

All saliva samples were frozen at $-20{ }^{\circ} \mathrm{C}$ immediately on arrival at the laboratory and were subsequently analyzed by radioimmunoassay (RIA), using a cortisol Coat-A-Count kit (DPC-Siemens Medical Solutions Diagnostics) which has a $1.0 \mathrm{nmol} / \mathrm{L}$ sensitivity and morning reference values of between 1.38 and $57.66 \mathrm{nmol} / \mathrm{L}$. All samples were analyzed in duplicate, and the samples of the same subject were included in the same assay. Although the variation coefficient necessary for replication was set at $8 \%$, the maximum intra- and inter-assay variation coefficients obtained were 4.3 and 5.2, respectively.

\section{Care Status}

Caregivers' feelings of burden were evaluated using the Zarit Burden Inventory (ZBI) created by Zarit et al. (1980). This instrument is composed of 22 items ranked on a five-point Likert scale from 0 (never) to 4 (nearly always) with a maximum score of 88 . The reliability coefficient is 0.92 . The items are related to health, social and personal life, and interpersonal relationships in the context of caring for individuals with functional and behavioral problems. Higher scores indicate a higher burden.

The degree of autism of the care recipient was also assessed; for this, we used the Autism Spectrum Quotient (AQ) developed by Baron-Cohen et al. (2006). This questionnaire was answered by caregivers and is composed of 50 items ranged on a four-point Likert scale with a reliability coefficient higher than 0.76. A higher score indicates a higher degree of autism with a maximum of 50 .

\section{Data Analysis}

For the analysis of the frequencies of the socio-demographic variables, chi-squared statistics were used. Mann-Whitney $U$ tests were performed to explore differences between caregivers and non-caregivers in demographic and anthropometric variables (age and body mass index) and factors potentially related to cortisol levels (medication, alcohol, tobacco, and psychoactive substance consumption before saliva collection). Due to the small sample size, non-parametric Friedman tests were conducted in order to analyze differences in afternoon Csal levels from pre-session to 0,15 , and $30 \mathrm{~min}$ post-session. This type of analysis was also employed to analyze differences in self-reported health measures between pre-, mid-, and post-treatment. Post hoc analyses were conducted with the Wilcoxon signed-rank test, with Bonferroni adjustments for multiple comparisons. The Wilcoxon signedrank test was also employed to analyze differences between pre- and post-session scores on the mood questionnaires. For self-reported health and mood profiles, change scores were calculated: in the case of self-reported health, change scores were calculated as the scores post-treatment minus those pretreatment, while change scores for mood profiles were calculated as the scores post-session minus those pre-session. The magnitude of the afternoon Csal response was estimated by the area under the curve with respect to the increase (AUCi), which was calculated using the trapezoid formula as previously described (Pruessner et al. 2003). The Mann-Whitney $U$ test was also employed to analyze differences between groups in the AUCi of Csal and change scores. Data analyses were carried out using SPSS 21.0 software (IBM SPSS), and $p$ values $\leq 0.05$ were considered significant. Values in the tables are expressed as mean $\pm \mathrm{SD}$.

\section{Results}

No differences were found between groups in sociodemographic variables. Descriptive data for all participants and for each group are summarized in Table 2 .

With regard to the CAR, Csal responses from awakening to $30 \mathrm{~min}$ later for all participants were $3.80 \mathrm{nmol} / \mathrm{L}$ in the pretreatment, $5.07 \mathrm{nmol} / \mathrm{L}$ in the mid-treatment, and $3.32 \mathrm{nmol} / \mathrm{L}$ in the post-treatment measurements. All of the responses were within the range proposed as normal $(2.5 \mathrm{nmol} / \mathrm{L})$, and no differences were found between pre-, mid-, and post-treatment.

\section{Does Each Evaluated MBP Session Affect Mood Disturbances and Csal? Are the Effects More Pronounced in Caregivers?}

In order to analyze the effectiveness of the evaluated sessions of the program (pre-, mid-, and post-treatment) in reducing mood disturbances and Csal levels, analyses were conducted with the whole sample and for each group separately. 
Table 2 Mean and standard deviation (SD) and frequency and percentage in demographic characteristics in caregivers, non-caregivers, and total sample

\begin{tabular}{|c|c|c|c|c|}
\hline Variable/characteristics & & Caregivers $(N=6)$ & Non-caregivers $(N=7)$ & Total sample $(N=13)$ \\
\hline \multirow[t]{2}{*}{ Gender } & Male & $1(16.7 \%)$ & $0(0 \%)$ & $1(7.7 \%)$ \\
\hline & Female & $5(83.3 \%)$ & $7(100 \%)$ & $12(92.3 \%)$ \\
\hline Age & & $44.33 \pm 5.24$ & $48 \pm 2.70$ & $46.30 \pm 4.32$ \\
\hline Body mass index (BMI) & & $25.48 \pm 6.65$ & $24.24 \pm 5.21$ & $24.81 \pm 5.69$ \\
\hline \multirow[t]{3}{*}{ Phases of the menstrual cycle } & Luteal & $4(80 \%)$ & $2(28.6 \%)$ & $6(46.2 \%)$ \\
\hline & Follicular & $0(0 \%)$ & $2(28.6 \%)$ & $2(15.4 \%)$ \\
\hline & Amenorrhea & $1(20 \%)$ & $3(42.9 \%)$ & $4(30.8 \%)$ \\
\hline \multirow[t]{2}{*}{ Marital status } & Married & $6(100 \%)$ & $4(66.7 \%)$ & $10(83.3 \%)$ \\
\hline & Widowed & $0(0 \%)$ & $2(33.3 \%)$ & $2(16.7 \%)$ \\
\hline \multirow[t]{3}{*}{ Educational level } & Basic & $1(16.7 \%)$ & $0(0 \%)$ & $1(8.3 \%)$ \\
\hline & Advanced & $1(16.7 \%)$ & $0(0 \%)$ & $1(8.3 \%)$ \\
\hline & University & $4(66.7 \%)$ & $6(100 \%)$ & $10(83.3 \%)$ \\
\hline \multirow[t]{3}{*}{ Source of income } & Pension & $1(16.7 \%)$ & $0(0 \%)$ & $1(8.3 \%)$ \\
\hline & Job & $3(50 \%)$ & $6(100 \%)$ & $9(75 \%)$ \\
\hline & Others & $2(33.3 \%)$ & $0(0 \%)$ & $2(16.7 \%)$ \\
\hline \multirow[t]{2}{*}{ Use of cigarettes } & Yes & $2(33.3 \%)$ & $0(0 \%)$ & $2(16.7 \%)$ \\
\hline & No & $4(66.7 \%)$ & $6(100 \%)$ & $10(83.3 \%)$ \\
\hline \multicolumn{5}{|l|}{ Care status } \\
\hline \multicolumn{2}{|l|}{ Years of evolution of care } & \multicolumn{3}{|l|}{$10 \pm 6.98$} \\
\hline \multicolumn{2}{|l|}{ Time weekly caring (h) } & \multicolumn{3}{|l|}{$30.44 \pm 34.92$} \\
\hline \multicolumn{2}{|l|}{ Care burden index } & \multicolumn{3}{|l|}{$49.33 \pm 21.15$} \\
\hline \multicolumn{5}{|l|}{ Characteristics of patient } \\
\hline \multirow[t]{2}{*}{ Diagnosis of patient } & Autism & \multicolumn{3}{|l|}{$4(66.7 \%)$} \\
\hline & Asperger & \multicolumn{3}{|l|}{$2(33.3 \%)$} \\
\hline \multirow[t]{2}{*}{ Gender } & Male & \multicolumn{3}{|l|}{$4(66.7 \%)$} \\
\hline & Female & \multicolumn{3}{|l|}{$2(33.3 \%)$} \\
\hline \multicolumn{2}{|l|}{ Age } & \multicolumn{3}{|l|}{$15 \pm 6.48$} \\
\hline \multicolumn{2}{|l|}{ Autism quotient (AQ) } & \multicolumn{3}{|l|}{$29.83 \pm 5.26$} \\
\hline
\end{tabular}

Analyzing the whole sample, significant changes were found in afternoon Csal from pre- to post-session at all time points evaluated $\left[\chi^{2}(3)=27.324, p=0.0001 ; \chi^{2}(3)=30.879\right.$, $p=0.0001$; and $\chi^{2}(3)=21.000, p=0.0001$, pre-, mid-, and post-treatment, respectively]. Post hoc analysis was conducted using the Wilcoxon signed-rank tests with Bonferroni adjustments applied, considering results significant at $p<0.008$. There were significant differences between Csal levels presession and $0 \mathrm{~min}$ post-session $(Z=-2.903, p=0.004$; $Z=-3.110, p=0.002$; and $Z=-3.041, p=0.002$, pre-, mid-, and post-treatment, respectively), $15 \mathrm{~min}$ post-session $(Z=-3.059, p=0.002 ; Z=-3.180, p=0.001 ;$ and $Z=-3.110$, $p=0.002$, pre-, mid-, and post-treatment), and 30 min postsession $(Z=-3.061, p=0.002 ; Z=-3.180, p=0.001$; and $Z=-2.970, p=0.003$, pre-, mid-, and post-treatment, respectively). In all cases, Csal levels were higher pre-session than 0 , 15 , and 30 min post-session. Regarding mood profiles, significant changes from pre- to post-session were found in the pretreatment session for anxiety $(\mathrm{Z}=-2.944, p=0.003)$, feelings of anger $(Z=-2.070, p=0.038)$, depression $(Z=-2.032$, $p=0.42)$, cholera $(Z=-2.060, p=0.039)$, fatigue $(Z=-3.101)$, tension $(Z=-2.321, p=0.020)$, and total $\operatorname{mood}(Z=-2.516$, $p=0.012$ ). Mid-treatment, changes from pre- to post-session were found for anxiety $(Z=-2.121, p=0.034)$, feelings of anger $(Z=-2.121, p=0.034)$, fatigue $(Z=-2.657, p=0.008)$, tension $(Z=-2.532, p=0.011)$, and total $\operatorname{mood}(Z=-2.345$, $p=0.019)$. Finally, post-treatment, changes were found in cholera $(Z=-2.060, p=0.039)$, fatigue $(Z=-1.992$, $p=0.046)$, and total $\operatorname{mood}(Z=-2.555, p=0.011)$. For all of the evaluated variables, post-session scores were lower than pre-session scores.

As noted above, the same analyses were conducted in each group separately. For caregivers, differences in afternoon Csal were found for all evaluated sessions $\left[\chi^{2}(3)=15.235\right.$, $p=0.002 ; \chi^{2}(3)=14.667, p=0.002$; and $\chi^{2}(3)=13.881$, $p=0.003$, pre-, mid-, and post-treatment, respectively]. However, no significant effects were found in the post hoc analyses. In the case of mood profiles, pre-treatment, there 
were reductions in anxiety $(Z=-2.201, p=0.028)$, fatigue $(Z=-2.226, p=0.026)$, tension $(Z=-2.023, p=0.043)$, and total negative $\operatorname{mood}(Z=-2.023, p=0.043)$, while reductions were found mid-treatment in anxiety $(Z=-2.060, p=0.039)$, tension $(Z=-2.214, p=0.027)$, and total negative mood $(Z=-1.997, p=0.043)$ and post-treatment session only in anxiety $(Z=-2.201, p=0.028)$. For non-caregivers, differences in afternoon Csal were also found for all sessions evaluated $\left[\chi^{2}(3)=12.176, p=0.007 ; \chi^{2}(3)=16.355, p=0.001\right.$; and $\chi^{2}$ $(3)=8.732, p=0.033$, pre-, mid-, and post-treatment, respectively], but again, differences did not reach significance in the post hoc analyses. For mood profiles, anxiety $(Z=-1.992$, $p=0.046)$ and fatigue $(Z=-2.226, p=0.026)$ showed a significant reduction in the pre-treatment session and only anxiety also showed a significant reduction post-treatment $(Z=-2.201, p=0.028)$, while no significant changes were found in any mood state mid-treatment (Fig. 1).

With the aim of comparing the magnitude of the reductions in negative mood and afternoon Csal between caregivers and non-caregivers, change scores for mood profiles and AUCs of the afternoon Csal levels were compared between groups. No differences were found in any of the mood profile change scores. On the other hand, differences were found in AUCs of the afternoon Csal levels post-treatment $(U=5, p=0.022)$, with caregivers presenting a greater reduction in Csal than non-caregivers (Fig. 2).

\section{Does the Whole MBP Program Affect Health? Are the Effects More Pronounced in Caregivers?}

As in the previous case, differences in self-reported health between evaluated sessions have been analyzed in the whole sample and for each group separately. In the total sample, significant changes were found in depressive symptomatology $\left[\chi^{2}(2)=15.854, p=0.0001\right]$. Again, post hoc analysis was conducted using the Wilcoxon signed-rank tests with

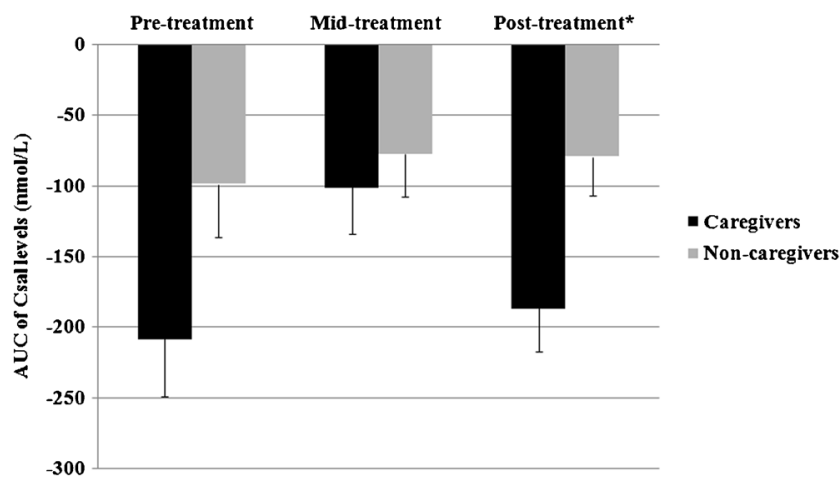

Fig. 2 AUC of afternoon Csal levels in pre, mid and post-treatment for caregivers and non-caregivers. ${ }^{*} p<.05$

Bonferroni adjustments applied, in this case $p<0.016$ being considered significant. There were significant differences between pre- and mid-treatment $(Z=-2.538, p=0.011)$ and between pre- and post-treatment $(Z=-2.937, p=0.003)$, with higher rates of depressive symptomatology pre-treatment than either mid- or post-treatment.

Regarding somatic symptoms, differences were found in immunological $\left[\chi^{2}(2)=6.682, p=0.035\right]$, cardiovascular $\left[\chi^{2}(2)=8.667, p=0.035\right]$, gastrointestinal $\left[\chi^{2}(2)=12.400\right.$, $p=0.002]$, neurosensory $\left[\chi^{2}(2)=6.711, p=0.035\right]$, muscular $\left[\chi^{2}(2)=16.044, p=0.0001\right]$, dermatological $\left[\chi^{2}(2)=15.116\right.$, $p=0.001]$, female reproductive system $\left[\chi^{2}(2)=13.613\right.$, $p=0.001]$, and total $\left[\chi^{2}(2)=14.596, p=0.001\right]$ symptoms. Post hoc analysis showed differences between pre- and midtreatment for gastrointestinal $(Z=-2.398, p=0.016)$, muscular $(Z=-2.762, p=0.006)$, neurosensory $(Z=-2.555, p=0.11)$, dermatological $(Z=-2.946, p=0.003)$, and total $(Z=-2.491$, $p=0.013)$ symptoms. Differences between pre- and posttreatment were found in muscular $(Z=-3.065, p=0.002)$, gastrointestinal $(Z=-2.807, p=0.005)$, dermatological $(Z=-2.823, p=0.005)$, female reproductive system $(Z=-2.536, p=0.011)$, and total $(Z=-2.981, p=0.003)$
Fig. 1 Negative mood (POMS Total Score) pre and post session in pre, mid and post-treatment for caregivers and noncaregivers

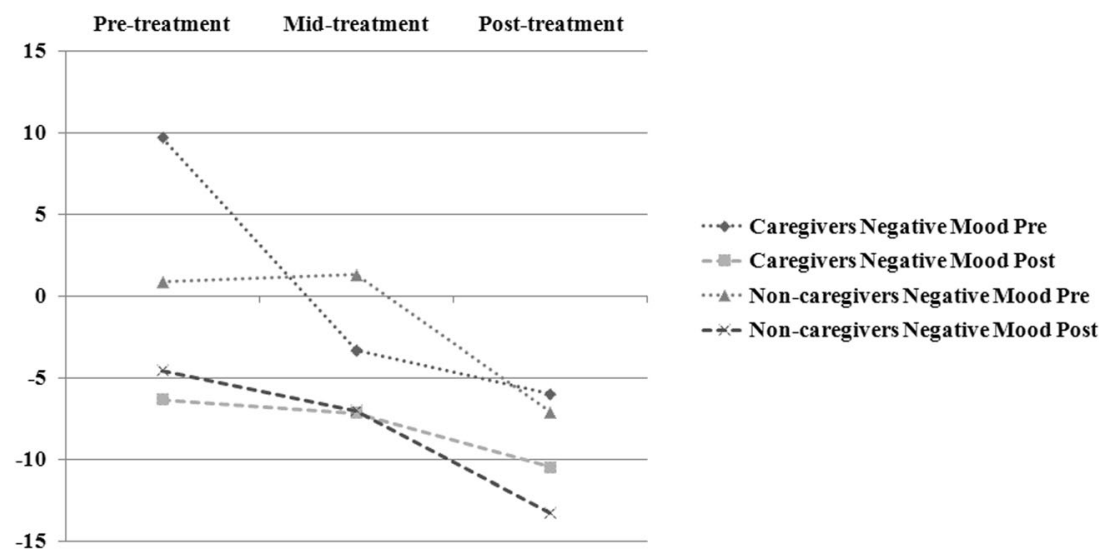


symptoms. Only in the case of cardiovascular $(Z=-2.588$, $p=0.010)$ and total $(Z=-2.551, p=0.011)$ symptoms were differences also found between mid- and post-treatment. For all of the symptoms evaluated, participants reported higher levels of symptoms pre-treatment than either mid- or posttreatment. In the case of cardiovascular and total symptoms, participants also reported higher levels of symptoms midtreatment than post-treatment.

For self-perceived general health, significant differences were observed in somatic symptoms $\left[\chi^{2}(2)=14.609\right.$, $p=0.001]$, anxiety and insomnia $\left[\chi^{2}(2)=18.426, p=0.000\right]$, social dysfunction $\left[\chi^{2}(2)=15.048, p=0.001\right]$, and severe depression $\left[\chi^{2}(2)=6.333, p=0.042\right]$. Post hoc analyses identified differences between pre- and mid-treatment in somatic symptoms $(Z=-2.556, p=0.011)$, anxiety and insomnia $(Z=-3.186, p=0.001)$, social dysfunction $(Z=-2.824$, $p=0.005)$, and self-perceived general health $(Z=-2.812$, $p=0.005)$. Differences were also found between pre- and post-treatment in somatic symptoms $(Z=-3.076, p=0.002)$, anxiety and insomnia $(Z=-3.045, p=0.002)$, social dysfunction $(Z=-2.814, p=0.005)$, and self-perceived general health $(Z=-3.045, p=0.002)$.

Conducting the same analyses in caregivers alone, differences were found in depressive symptomatology $\left[\chi^{2}(2)=10.182, p=0.006\right]$, as well as in immunological $\left[\chi^{2}\right.$ $(2)=6.091, p=0.048]$, cardiovascular $\left[\chi^{2}(2)=5.818\right.$, $p=0.050]$, gastrointestinal $\left[\chi^{2}(2)=6.636, p=0.036\right]$, neurosensory $\left[\chi^{2}(2)=7.636, p=0.022\right]$, muscular $\left[\chi^{2}(2)=7.000\right.$, $p=0.030]$, dermatological $\left[\chi^{2}(2)=9.238, p=0.010\right]$, and female reproductive system $\left[\chi^{2}(2)=8.000, p=0.018\right]$ symptoms. In the case of self-perceived general health, significant differences were found in somatic symptoms $\left[\chi^{2}(2)=7.000\right.$, $p=0.030]$, anxiety and insomnia $\left[\chi^{2}(2)=6.522, p=0.038\right]$, and social dysfunction $\left[\chi^{2}(2)=9.333, p=0.009\right]$. For noncaregivers, differences were found in depressive symptomatology $\left[\chi^{2}(2)=6.000, p=0.05\right]$, as well as gastrointestinal $\left[\chi^{2}(2)=7.630, p=0.022\right]$, muscular $\left[\chi^{2}(2)=10.320\right.$, $p=0.006]$, dermatological $\left[\chi^{2}(2)=7.923, p=0.019\right]$, female reproductive system $\left[\chi^{2}(2)=7.111, p=0.029\right]$, and total $\left[\chi^{2}\right.$ $(2)=11.308, p=0.004]$ symptoms. Factors of self-perceived general health, namely somatic symptoms $\left[\chi^{2}(2)=9.818\right.$, $p=0.007]$, anxiety and insomnia $\left[\chi^{2}(2)=12.333, p=0.002\right]$, social dysfunction $\left[\chi^{2}(2)=6.333, p=0.042\right]$, and selfperceived general health $\left[\chi^{2}(2)=11.083, p=0.004\right]$ also showed significant differences. However, no significant differences were found in post hoc analyses in either of the two groups (Table 3).

As with mood states, change scores for self-reported health measures were compared between caregivers and noncaregivers. Change scores for respiratory $(U=5, p=0.041)$, neurosensory $(U=3, p=0.015)$, and total $(U=7, p=0.05)$ symptoms were different between groups, being higher in caregivers than non-caregivers.

\section{Discussion}

Our results suggest the value of the MBP as an effective intervention for reducing health complaints and mood disturbances, in both caregivers and non-caregivers. Specifically, there were lower levels of depressive and somatic symptoms after the program, showing a progressive reduction during the intervention. With this progressive reduction, participants' self-perceived general health improved. The whole program had a positive effect on health in all participants, and each session evaluated independently reduced anxiety, negative mood, and Csal as well-although this effect was more pronounced in caregivers. Specifically, the fall in Csal levels during the post-treatment session was more marked in caregivers than in non-caregivers. In this regard, caregivers could be more sensitive to the effects of the exercises and meditation practiced during the sessions. This would then explain the greater reduction in afternoon Csal levels in this group. Previous research has demonstrated that caregivers of people with ASDs have high daily levels of negative emotions (Smith et al. 2010), and these are mainly explained by challenges associated with the care situation (Ludlow et al. 2012; De Andrés-García et al. 2013). In this context, meditation and the types of exercises carried out during the sessions seem to significantly reduce Csal levels and mood disturbances. As noted by other authors, cortisol is a reliable and sensitive indicator of the influence of meditation on the functioning of the hypothalamic-pituitary-adrenal axis (Matousek et al. 2010). For this reason, our results seem to reinforce the idea that caregivers are more sensitive to the MBP sessions than the general non-chronically stressed population. Summative explanations could be related to the greater motivation and implication of caregivers in the MBP program, which probably affects the marked reduction in afternoon Csal levels observed in caregivers.

With regard to self-reported health, depressive and somatic symptoms diminished markedly over the program, especially in caregivers, who showed greater reductions in respiratory, neurosensory, and total somatic symptoms. Together with these specific reports, participants had better self-perceived general health after finishing the program. In accordance with these results, recent studies have demonstrated that mindfulness interventions are effective for reducing depressive symptomatology and health complaints in clinical and healthy populations (Keng et al. 2011; Klainin-Yobas et al. 2012). The underlying mechanism for explaining these effects is unclear; nevertheless, several explanations have been suggested (Hölzel et al. 2011). Specifically, training mindfulness by an intervention program could increase the mindfulness trait in individuals through the effects of continuous meditation and exercise (Kabat-Zinn 2005; Keng et al. 2011). In relation to this, some authors have proposed that an increase in trait mindfulness is beneficial for chronic conditions, such as 
Table 3 Scores (mean and standard deviation) in self-reported health (depressive symptomatology, somatic symptoms, and perceived general health) in pre, mid, and post-treatment for caregivers and non-caregivers separately

\begin{tabular}{|c|c|c|c|c|c|c|}
\hline & \multicolumn{2}{|l|}{ Pre-treatment } & \multicolumn{2}{|l|}{ Mid-treatment } & \multicolumn{2}{|c|}{ Post-treatment } \\
\hline & Caregivers & Non-caregivers & Caregivers & Non-caregivers & Caregivers & Non-caregivers \\
\hline Depressive symptomatology & $10.33 \pm 5.35$ & $7.85 \pm 9.37$ & $3.83 \pm 2.85$ & $2.42 \pm 3.40$ & $3.33 \pm 3.32$ & $0.71 \pm 0.75$ \\
\hline \multicolumn{7}{|l|}{ Somatic symptoms } \\
\hline Immunological & $11.50 \pm 5.31$ & $6.50 \pm 4.72$ & $5.33 \pm 3.07$ & $5 \pm 4.09$ & $2.83 \pm 1.94$ & $4.16 \pm 4.62$ \\
\hline Respiratory & $13.16 \pm 4.99$ & $6.16 \pm 3.43$ & $6 \pm 4.14$ & $5.66 \pm 4.45$ & $4.50 \pm 4.96$ & $5.66 \pm 7.55$ \\
\hline Cardiovascular & $8 \pm 4.93$ & $3.16 \pm 2.99$ & $4.16 \pm 5.49$ & $3.50 \pm 3.39$ & $2 \pm 3.52$ & $1.83 \pm 2.85$ \\
\hline Gastrointestinal & $15.83 \pm 7.49$ & $10.16 \pm 10.96$ & $9 \pm 4$ & $5 \pm 4.69$ & $6.33 \pm 5.88$ & $5 \pm 6.78$ \\
\hline Neurosensory & $16.16 \pm 5.77$ & $5.33 \pm 3.07$ & $7.16 \pm 6.73$ & $3.50 \pm 3.39$ & $6.66 \pm 6.12$ & $4.83 \pm 5.03$ \\
\hline Genital-urinary & $13 \pm 5.44$ & $4.83 \pm 5.11$ & $5.33 \pm 6.31$ & $6.50 \pm 7.39$ & $3.83 \pm 5.56$ & $3.83 \pm 4.30$ \\
\hline Muscular & $20.33 \pm 4.27$ & $13.33 \pm 7.99$ & $9.33 \pm 8.18$ & $10 \pm 8.29$ & $8 \pm 7.15$ & $6.66 \pm 4.08$ \\
\hline Dermatological & $11.83 \pm 4.95$ & $6.33 \pm 7.73$ & $4.50 \pm 4.63$ & $3.50 \pm 6.65$ & $4.83 \pm 5.45$ & $5.16 \pm 7.54$ \\
\hline Female reproductive & $13.33 \pm 10.50$ & $14.50 \pm 8.06$ & $6.33 \pm 6.77$ & $10 \pm 7.77$ & $4 \pm 6.03$ & $6.33 \pm 6.34$ \\
\hline Total symptoms & $123.16 \pm 28.75$ & $70.33 \pm 34.93$ & $57.16 \pm 35.14$ & $52.66 \pm 31.42$ & $43 \pm 31.65$ & $43.50 \pm 37.90$ \\
\hline \multicolumn{7}{|l|}{ Perceived general health } \\
\hline Somatic symptoms & $6.33 \pm 3.72$ & $6.28 \pm 5.15$ & $3.66 \pm 2.94$ & $2.85 \pm 2.19$ & $1.66 \pm 2.06$ & $2.57 \pm 1.71$ \\
\hline Anxiety and insomnia & $6.50 \pm 4.03$ & $8.42 \pm 5.41$ & $1.50 \pm 0.83$ & $1.85 \pm 2.03$ & $1.16 \pm 1.16$ & $1.85 \pm 2.19$ \\
\hline Social dysfunction & $5.33 \pm 3.38$ & $6.42 \pm 2.99$ & $3 \pm 2$ & $4.28 \pm 2.56$ & $1.83 \pm 1.72$ & $3.71 \pm 1.97$ \\
\hline Severe depression & $1.16 \pm 1.60$ & $2.57 \pm 4.31$ & $0.16 \pm 0.40$ & $0.14 \pm 0.37$ & $0.16 \pm 0.40$ & $0.28 \pm 0.48$ \\
\hline Total perceived health & $10.66 \pm 4.45$ & $11 \pm 7.32$ & $5.16 \pm 2.56$ & $5.28 \pm 3.77$ & $3.33 \pm 3.14$ & $5.14 \pm 3.97$ \\
\hline
\end{tabular}

depression or chronic diseases (Ghasemipour et al. 2013; Keng et al. 2011). Although there is no consensus in the literature about the definition of trait mindfulness, Brown and Ryan (2003) defined this trait as "the general tendency of individuals to be attentive to and aware of experiences in daily life." Previous correlational studies have found this trait to be related positively to vitality and positive affect (Brown and Ryan 2003) and negatively to depression, negative affect, and difficulties in emotional regulation (Baer et al. 2006; Cash and Whittingham 2010; Giluk 2009). Furthermore, experimental studies have found that trait mindfulness operates as a mediator between the effectiveness of mindfulness intervention and positive outcomes (Keng et al. 2011). One of the most important factors in caregivers could be living in the present moment with non-judgmental acceptance, which is closely related to low levels of rumination and worry as it blocks the mental processes of anticipation. Some authors have suggested that this mechanism may operate in caregivers of people with dementia, taking into account that they could be involved in rumination processes related to past events before the disease and events in the future related to the course of the dementia. This last issue could be especially relevant in caregivers of people with ASDs, since one of the greatest worries of this population is the future of their affected offspring (Phelps et al. 2009).
Training in acceptance, another component of MBP, teaches caregivers to accept the situation and the chronic character of their offspring's developmental disability. Acceptance allows the situation to be perceived anew, as well as enables positive reappraisal and non-reactivity to inner experiences (Shapiro et al. 2006). Helping individuals to discuss and find different ways of coping with the daily challenges associated with the care situation could promote alternative views of this situation and ways of dealing with it. In a care context, this would be an essential mechanism for reducing stress and improving well-being through reappraisal processes. This could be proposed as another mechanism to explain the efficacy of this type of intervention as it enables caregivers to regulate negative emotions and develop adaptive coping processes.

This preliminary study entails an advance in the comprehension of the effects of MBP interventions on general health, as it includes biological markers together with self-perceived measures. Nevertheless, some limitations must be addressed. The main limitations are the sample size and the lack of a waiting list control design, fundamentally in the case of caregivers, which limit our ability to draw conclusions about causality. Alternative explanations of the greater reduction in Csal levels and somatic symptoms in caregivers could involve a manifestation of the regression to the mean effect (Barnett et al. 2005). This effect is especially relevant in repeated 
measures analysis in samples with baseline scores extremely different from the population mean. In this case, caregivers are at risk of presenting higher initial levels of symptoms and Csal, due to their stress condition, compared to noncaregivers. In this sense, this effect would explain participants with high levels of symptoms at baseline generally improving more than those with low symptoms. Hence, the greater reductions observed in the caregivers could be explained by this phenomenon, resulting in a statistical tendency of the initial scores in caregivers to approach the mean. Future studies with larger numbers of caregivers and controls would allow other types of statistical analysis to be conducted, avoiding these confounding effects.

Moreover, no measures of trait mindfulness have been evaluated. It would also be interesting to analyze the effects of the program on this trait in future research. The fact that only the experimental group included a man, the controls all being women, could also influence the results obtained, and the possible differences between genders in the effectiveness of MBP have not been controlled for in the analysis. Another limitation is the lack of burden measures after the MBP program, even though previous research has found a mindfulness intervention to have little effect on burden (Whitebird et al. 2012).

On the other hand, the wide range of variables evaluated (including biological markers of health) and the high rate of attendance and adequate response of the participants during the program provide this study with a high level of validity to assess the effectiveness of the MBP intervention. These latter factors are notable as caregivers typically lack time and have difficulties in following an established timetable for participating in any intervention. Overall, we consider that these factors make the data that we have obtained particularly valuable.

\section{References}

Baer, R. A., Smith, G. T., Hopkins, J., Krietemeyer, J., \& Toney, L. (2006). Using self-report assessment methods to explore facets of mindfulness. Assessment, 13(1), 27-45.

Barnett, A. G., van de Pols, J. C., \& Dobson, A. J. (2005). Regression to the mean: what it is and how to deal with it. International Journal of Epidemiology, 34(1), 215-220.

Baron-Cohen, S., Hoekstra, R. A., Knickmeyer, R., \& Wheelwright, S. (2006). The autism spectrum quotient (AQ)-adolescent version. Journal of Autism and Developmental Disorders, 36(3), 343-350.

Beck, A. T., \& Steer, R. A. (1993). Beck depression inventory manual. San Antonio: The Psychological Corporation.

Birnie, K., Garland, S. N., \& Carlson, L. E. (2010). Psychological benefits for cancer patients and their partners participating in mindfulness-based stress reduction (MBSR). Psychooncology, 19(9), 1004-1009.
Bränström, R., Kvillemo, P., \& Akerstedt, T. (2013). Effects of mindfulness training on levels of cortisol in cancer patients. Psychosomatics, 54(2), 158-164.

Brown, K. W., \& Ryan, R. M. (2003). The benefits of being present: mindfulness and its role in psychological well-being. Journal of Personality and Social Psychology, 84, 822-848.

Carlson, L. E., Speca, M., Faris, P., \& Patel, K. D. (2007). One year prepost intervention follow-up of psychological, immune, endocrine and blood pressure outcomes of mindfulness-based stress reduction (MBSR) in breast and prostate cancer outpatients. Brain, Behavior, and Immunity, 21(8), 1038-1049.

Cash, M., \& Whittingham, K. (2010). What facets of mindfulness contribute to psychological well-being and depressive, anxious, and stress-related symptomatology? Mindfulness, 1(1), 177-182.

De Andrés-García, S., Moya-Albiol, L., \& González-Bono, E. (2012). Salivary cortisol and immunoglobulin A: responses to stress as predictors of health complaints reported by caregivers of offspring with autistic spectrum disorder. Hormones and Behavior, 62(4), 464-474.

De Andrés-García, S., Sariñana-González, P., Romero-Martínez, A., Moya-Albiol, L., \& González-Bono, E. (2013). Cortisol responses to stress in caregivers of offspring with autism spectrum disorder is associated with care recipient characteristics. Stress, 16(5), 510-519.

Dickmeis, T. (2009). Glucocorticoids and the circadian clock. Journal of Endocrinology, 200(1), 3-22.

Ferraioli, S. J., \& Harris, S. L. (2012). Comparative effects of mindfulness and skills based parent training programs for parents of children with autism: feasibility and preliminary outcome data. Mindfulness, 4(2), 89-101.

Franco, C., Sola, M. M., \& Justo, E. (2010). Reducción del malestar psicológico y de la sobrecarga en familiares cuidadores de enfermos de Alzheimer mediante la aplicación de un programa de entrenamiento en Mindfulness (conciencia plena). Revista Española de Geriatría y Gerontología, 45(5), 252-258.

Fuentes, I., Balaguer, I., Meliá, J., \& García-Merita, M. (1995). Forma abreviada del Perfil de Estados de Ánimo (POMS). In E. Cantó n (Ed.), Libro de Actas del V Congreso Nacional de Psicologia de la Actividad Física y el Deporte. Valencia: Universitat de Valéncia.

Ghasemipour, Y., Robinson, J.A., \& Ghorbani, N. (2013). Mindfulness and integrative self-knowledge: relationships with health-related variables. International Journal of Psychology.

Giluk, T. L. (2009). Mindfulness, big five personality, and affect: a metaanalysis. Personality and Individual Differences, 47(8), 805-811.

Goldberg, D. P., \& Hillier, V. (1979). A scaled version of the general health questionnaire. Psychological Medicine, 9(1), 139-145.

Goodman, M. J., \& Schorling, J. B. (2012). A mindfulness course decreases burnout and improves well-being among healthcare providers. International Journal of Psychiatry in Medicine, 43(2), 119 128.

Hölzel, B. K., Lazar, S. W., Gard, T., Schuman-Olivier, Z., Vago, D. R., \& Ott, U. (2011). How does mindfulness meditation work? Proposing mechanisms of action from a conceptual and neural perspective. Perspectives on Psychological Science, 6(6), 537-559.

Kabat-Zinn, J. (2005). Coming to our senses. London: Piatkus Books Ltd.

Keng, S. L., Smoski, M. J., \& Robins, C. J. (2011). Effects of mindfulness on psychological health: a review of empirical studies. Clinical Psychology Review, 31(6), 1041-1056.

Kirkham, M. A. (1993). Two-year follow-up of skills training with mothers of children with disabilities. American Journal of Mental Retardation, 97(5), 509-520.

Klainin-Yobas, P., Cho, M. A., \& Creedy, D. (2012). Efficacy of mindfulness-based interventions on depressive symptoms among people with mental disorders: a meta-analysis. International Journal of Nursing Studies, 49(1), 109-121.

Lengacher, C. A., Kip, K. E., Barta, M. K., Post-White, J., Jacobsen, P., Groer, M., et al. (2012). A pilot study evaluating the effect of 
mindfulness-based stress reduction on psychological status, physical status, salivary cortisol, and interleukin-6 among advanced-stage cancer patients and their caregivers. Journal of Holistic Nursing, 30(7), 170-185.

Ludlow, A., Skelly, C., \& Rohleder, P. (2012). Challenges faced by parents of children diagnosed with autism spectrum disorder. Journal of Health Psychology, 17(5), 702-711.

Lykins, E. L. B., \& Baer, R. A. (2009). Psychological functioning in a sample of long-term practitioners of mindfulness meditation. Journal of Cognitive Psychotherapy, 23(3), 226-241.

Matousek, R., Dobkin, P. L., \& Pruessner, J. (2010). Cortisol as a marker for improvement in mindfulness-based stress reduction. Complementary Therapies in Clinical Practice, 16(1), 13-19.

Merkes, M. (2010). Mindfulness-based stress reduction for people with chronic diseases. Australian Journal of Primary Health, 16(3), 200 210.

Miguel-Tobal, J. J., Casado, M. I., Cano-Vindel, A., \& Spielberger, C. D. (2001). Inventario de Expresion de Ira Estado-Rasgo: STAXI-2. Madrid: TEA Ediciones.

Minor, H. G., Carlson, L. E., Mackenzie, M. J., Zernicke, K., \& Jones, L. (2006). Evaluation of a mindfulness-based stress reduction (MBSR) program for caregivers of children with chronic conditions. Social Work in Health Care, 43(1), 91-109.

Nixon, C. D., \& Singer, G. H. (1993). Group cognitive behavioral treatment for excessive parental self-blame and guilt. American Journal of Mental Retardation, 97(6), 665-672.

Oken, B. S., Fonareva, I., Haas, M., Wahbeh, H., Lane, J. B., Zajdel, D., et al. (2010). Pilot controlled trial of mindfulness meditation and education for dementia caregivers. Journal of Alternative and Complementary Medicine, 16(10), 1031-1038.

Pérez-Blasco, J., Viguer, P., \& Rodriguez, M. F. (2013). Effects of a mindfulness-based intervention on psychological distress, wellbeing and maternal self-efficacy in breastfeeding mothers: results of a pilot study. Archives of Women's Mental Health, 16(3), 227-236.

Phelps, K. W., Hodgson, J. L., McCammon, S. L., \& Lamson, A. L. (2009). Caring for an individual with autism disorder: a qualitative analysis. Journal of Intellectual and Developmental Disability, 34(1), 27-35.

Pruessner, J. C., Kirschbaum, C., Meinlschmid, G., \& Hellhammer, D. H. (2003). Two formulas for the computation of the area under the curve represent measures of total hormone concentration versus time-dependent change. Psychoneuroendocrinology, 28(7), 916931.
Ruiz-Robledillo, N., \& Moya-Albiol, L. (2013). Self-reported health and cortisol awakening response in parents of people with Asperger syndrome: the role of trait anger and anxiety, coping and burden. Psychology and Health, 28(11), 1246-1264.

Ruiz-Robledillo, N., González-Bono, E., \& Moya-Albiol, L. (2014). Lack of institutional support entails disruptions in cortisol awakening response in caregivers of people with high-functioning autism. Journal of Health Psychology.

Sandín, B., \& Chorot, P. (1995). Escala de Síntomas Somáticos-Revisada (ESS-R). Madrid: Universidad Nacional de Educación a Distancia (UNED).

Seisdedos, N. (1982). STAI: Inventario de Ansiedad Estado-Rasgo [State-Trait Anxiety Inventory]. Madrid: TEA Ediciones.

Shapiro, S. L., Carlson, L. E., \& Astin, J. A. (2006). Mechanisms of mindfulness. Journal of Clinical Psychology, 62, 373-386.

Singer, G., Ethridge, B., \& Aldana, S. (2007). Primary and secondary effects of parenting and stress management interventions for parents of children with developmental disabilities: a meta-analysis. Mental Retardation and Developmental Disabilities Research Reviews, 13(4), 357-369.

Singh, N. N., Lancioni, E., Winton, A. S. W., Fisher, B. C., Wahler, R. G., McAleavey, K., et al. (2006). Mindful parenting decreases aggression, noncompliance, and self-injury in children with autism. Journal of Emotional and Behavioral Disorders, 14(3), 169-177.

Singh, N. N., Lancioni, E., Winton, A. S. W., Singh, J., Curtis, W. J., Wahler, R. G., et al. (2007). Mindful parenting decreases aggression and increases social behavior in children with developmental disabilities. Behavior Modification, 31(6), 749-771.

Smith, L. E., Hong, J., Seltzer, M. M., Greenberg, J. S., Almeida, D. M., \& Bishop, S. L. (2010). Daily experiences among mothers of adolescents and adults with autism spectrum disorder. Journal of Autism and Developmental Disabilities, 40(2), 167-178.

Spielberger, C.D., Gorusch, R.L., Lushene, R., Vagg, P.R., \& Jacobs, G.A. (1983). Manual for the State-Trait Anxiety Inventory. Consulting Psychologists Press.

Whitebird, R., Kreitzer, M., Crain, A. L., Lewis, B. A., Hanson, L. H., \& Enstad, C. J. (2012). Mindfulness-based stress reduction for family caregivers: a randomized controlled trial. The Gerontologist, 53(4), 676-686.

Zarit, S. H., Reever, K. E., \& Bach-Peterson, J. (1980). Relatives of the impaired elderly: correlates of feelings of burden. Gerontologist, 20 , 649-654. 